\title{
The transient hard X-ray tail of GX 17+2: New BeppoSAX results
}

\author{
R. Farinelli ${ }^{1}$, F. Frontera ${ }^{1,2}$, A. A. Zdziarski ${ }^{3}$, L. Stella ${ }^{4}$, S. N. Zhang ${ }^{5,6}$, M. van der Klis ${ }^{7}$, N. Masetti ${ }^{2}$, and L. Amati ${ }^{2}$ \\ 1 Dipartimento di Fisica, Università di Ferrara, via Paradiso 12, 44100 Ferrara, Italy \\ e-mail: farinelli@fe.infn.it \\ 2 Istituto di Astrofisica Spaziale e Fisica Cosmica, Sezione di Bologna, CNR, via Gobetti 101, 40129 Bologna, Italy \\ ${ }^{3}$ N. Copernicus Astronomical Center, Bartycka 18, 00-716 Warsaw, Poland \\ 4 Osservatorio Astronomico di Roma, via Frascati 33, 00040 Monteporzio Catone, Italy \\ 5 Physics Department, University of Alabama in Huntsville, AL 35899 Huntsville, USA \\ ${ }^{6}$ Space Sciences Laboratory, NASA Marshall Space Flight Center, SD50, AL 35812 Huntsville, USA \\ 7 Astronomical Institute “Anton Pannekoek”, University of Amsterdam, Kruislaan 403, 1098 SJ Amsterdam, The Netherlands
}

Received 30 June 2004 / Accepted 17 December 2004

\begin{abstract}
We report on results of two BeppoSAX observations of the Z source GX 17+2. In both cases the source is in the horizontal branch of the colour-intensity diagram. The persistent continuum can be fit by two-component models consisting of a blackbody plus a Comptonization spectrum. With one of these models, two solutions for the blackbody temperature of both the observed and seed photons for Comptonization are equally accepted by the data. In the first observation, when the source is on the left part of the horizontal branch, we observe a hard tail extending up to $120 \mathrm{keV}$, while in the second observation, when the source moves towards right in the same branch, the tail is no longer detected. The hard ( $\approx 30 \mathrm{keV}) \mathrm{X}$-ray emission can be modeled either by a simple power-law with photon index $\Gamma \sim 2.8$, or assuming Comptonization of $\sim 1 \mathrm{keV}$ soft photons off a hybrid thermal plus non-thermal electron plasma. The spectral index of the non-thermal injected electrons is $p \sim 1.7$. The observation of hard X-ray emission only in the left part of the horizontal branch could be indicative of the presence of a threshold in the accretion rate above which the hard tail disappears. An emission line at $6.7 \mathrm{keV}$ with equivalent width $\sim 30 \mathrm{eV}$ is also found in both observations. We discuss these results and their physical implications.
\end{abstract}

Key words. stars: individual: GX 17+2 - stars: neutron - X-rays: binaries - accretion, accretion disks

\section{Introduction}

$\mathrm{Z}$ sources form the brightest class of galactic Low Mass X-ray Binaries (LMXBs), with X-ray luminosities typically in the range $\sim 0.5-1$ times the Eddington luminosity (where $L_{\mathrm{Edd}} \sim$ $1.5 M / M_{\odot} \times 10^{38} \mathrm{erg} \mathrm{s}^{-1}$ ). Their name arises from the pattern they trace in the colour-colour or hardness-intensity diagram (CD and HID, respectively) on typical time scales of days. The three regions which characterize the Z-track, from the top, are the horizontal branch (HB), the normal branch (NB) and the flaring branch (FB). Multifrequency observations of Sco X-1 (Vrtilek et al. 1991a; Hertz et al. 1992; Augusteijn et al. 1992) and Cyg X-2 (van Paradaijs et al. 1990; Vrtilek et al. 1990; Hasinger et al. 1990) have shown that the accretion rate $\dot{M}$ increasing as the sources move in the direction $\mathrm{HB} \rightarrow \mathrm{NB} \rightarrow$ FB is consistent with UV observations and some QPO models. Several two-component models have been proposed to fit the low energy $(\$ 20 \mathrm{keV}) \mathrm{X}$-ray spectra of $\mathrm{Z}$ sources, such as a simple or Comptonized blackbody (BB) plus a multicolour disk blackbody (DBB, the "Eastern Model", Mitsuda et al. 1984, 1989), a BB plus an unsaturated Comptonization spectrum $\left(F(E) \propto E^{-\Gamma} \exp \left(-E / E_{\mathrm{c}}\right)\right.$, the "Western Model",
White et al. 1986, 1988) and, more recently, a DBB plus the Comptonization model worked out by Titarchuk (1994; COMPTT in XSPEC, Piraino et al. 2002; Di Salvo et al. 2002). In recent years, transient hard X-ray tails extending up to $100 \mathrm{keV}$ have been discovered from the classical Z sources (GX 5-1, Asai et al. 1994; Cyg X-2, Frontera et al. 1998; Di Salvo et al. 2002; GX 17+2, Di Salvo et al. 2000, hereafter DS2000; Sco X-1, D'Amico et al. 2001; GX 349+2, Di Salvo et al. 2001) and from the peculiar Z source Cir X-1 (Iaria et al. 2001). All these hard tails have been fit with power-laws (PL, $F(E) \propto E^{-\Gamma}$ ) with photon index $\Gamma$ ranging from $\sim-1$ (Sco X-1, when the source was in the FB) to $\sim 3.3$ (Cir X-1). The time behaviour of the hard tail is complex and still not well understood. DS2000 observed a strong hard tail in GX $17+2$ when the source was on the left part of the HB; as the source moved on the right across the branch, an independent fit of the photon index and PL normalization was not possible, and fixing the photon index at 2.7 yielded an intensity of the PL that lowered (chance probability of F-test $\sim 10^{-4}$ ) until it disappeared at the HB-NB apex. In the case of Cyg X-2 (Di Salvo et al. 2002) the hard tail was observed when the source was in the HB, while in a later observation, when the source was in the NB, no evidence 
Table 1. Log of the two observations of GX $17+2$ with the on-source exposure time for each NFI.

\begin{tabular}{|c|c|c|c|c|c|c|c|c|}
\hline Obs. & Start time (UT) & End time (UT) & $\begin{array}{c}\text { LECS } \\
\mathrm{ks}\end{array}$ & $\begin{array}{c}\text { MECS } \\
\text { ks }\end{array}$ & $\begin{array}{c}\text { HPGSPC } \\
\text { ks }\end{array}$ & $\begin{array}{c}\text { PDS } \\
\text { ks }\end{array}$ & $\begin{array}{c}1.8-10 \mathrm{keV} \text { MECS } \\
\text { count rate }\left(\mathrm{s}^{-1}\right)\end{array}$ & $\begin{array}{c}13-200 \mathrm{keV} \text { PDS } \\
\text { count rate }\left(\mathrm{s}^{-1}\right)\end{array}$ \\
\hline 1 & 1997 Apr. 03 00:27:52 & 1997 Apr. 03 06:00:06 & 2.3 & 10.7 & 4.1 & 3.4 & 176 & 35 \\
\hline 2 & 1997 Apr. 21 02:29:00 & 1997 Apr. 21 06:13:55 & 1.7 & 6.5 & 2.4 & 2.3 & 190 & 24 \\
\hline
\end{tabular}

of hard X-ray emission was found. The hard tail of GX 5-1 (Asai et al. 1994) was detected when the source was in the NB, and decreased as the source moved to the FB. If $\dot{M}$ actually increases from $\mathrm{HB}$ to $\mathrm{FB}$, these results could be indicative of the presence of an anti-correlation between hard X-ray emission and accretion rate $\dot{M}$. However, the results of D'Amico et al. (2001) contrast with this scenario, as in Sco X-1 the hard tail was observed in all branches without any apparent correlation with the source position on the CD.

\section{1. $G \times 17+2$}

GX $17+2$ is one of strongest Galactic X-ray sources, and was detected in the sixties (e.g., Friedman et al. 1967). Simultaneous X-ray and radio observations, performed by White et al. (1978), did not show the presence of significant radio emission, but revealed correlated X-ray intensity and spectral variations similar to those already observed from Sco X-1. Observation of the source with the Gas Scintillation Proportional Counter (2-20 keV) on-board EXOSAT (White et al. 1986) provided an accurate determination of the source continuum spectrum, which was fit in the context of the Western model with best-fit parameters $k T_{\mathrm{bb}} \sim 1 \mathrm{keV}$, radius of the BB emitting sphere $R_{\mathrm{bb}} \sim 15 \mathrm{~km}, \Gamma \sim 0.6$ and $E_{\mathrm{c}} \sim 4.5 \mathrm{keV}$. Also an emission line at $\sim 6.5 \mathrm{keV}$ with equivalent width $(E W)$ of $\sim 110 \mathrm{eV}$ was detected. The data analysis of the same observation, but with the Medium Energy Proportional Counter Array, showed that the 1-30 keV source spectrum could be fit with a $\mathrm{BB}\left(k T_{\mathrm{bb}} \sim 1.2 \mathrm{keV}\right)$ plus the Comptonization model proposed by Sunyaev \& Titarchuk (1980), with electron temperature $k T_{\mathrm{e}} \sim 3 \mathrm{keV}$ and optical depth of the electron cloud of $\tau \sim 13$ (White et al. 1988). The column density along the source direction was determined by Vrtilek et al. (1991b) with Einstein $(0.2-20 \mathrm{keV})$, who found a value of $N_{\mathrm{H}} \sim 1.5 \times$ $10^{22} \mathrm{~cm}^{-2}$, assuming a simple BB $\left(k T_{\mathrm{bb}} \sim 1.5 \mathrm{keV}\right)$ as best-fit model. Hoshi \& Asaoka (1993) observed the source with Ginga in both the HB and the NB. By fitting the 1-20 keV X-ray spectrum with the first version of the Eastern model (BB + DBB), these authors found that the inner disk temperature and the projected radius of the DBB did not change significantly from the $\mathrm{NB}$ to the $\mathrm{HB}$, while the $\mathrm{BB}$ temperature gradually increased from to $2.1 \mathrm{keV}$ to $2.7 \mathrm{keV}$. DS2000 analyzed the $0.1-100 \mathrm{keV}$ $\mathrm{X}$-ray spectrum of GX $17+2$ with BeppoSAX, discovering a transient hard X-ray tail in addition to the persistent continuum. The latter was well described by a BB plus COMPTT model, with best-fit parameters $k T_{\mathrm{bb}} \sim 0.6 \mathrm{keV}, R_{\mathrm{bb}} \sim 40 \mathrm{~km}$, temperature of the seed photons to be Comptonized $k T_{\mathrm{s}} \sim 1 \mathrm{keV}$, electron temperature $k T_{\mathrm{e}} \sim 3 \mathrm{keV}$ and optical depth of the Comptonizing cloud $\tau \sim 10$, assuming a spherical geometry. Additionally, they found evidence for an emission line with energy centroid $E_{1} \sim 6.7 \mathrm{keV}$ and $E W \sim 35 \mathrm{eV}$, and an absorption edge with $E_{\text {edge }} \sim 8.6 \mathrm{keV}$ and optical depth $\tau_{\text {aedge }} \sim 3 \times 10^{-2}$. Type I X-ray bursts from GX 17+2 were observed with the Einstein (Kahn \& Grindlay 1984), HAKUCHO (Tawara et al. 1984) and EXOSAT (Sztajno et al. 1986) satellites.

Despite the X-ray brightness, the optical counterpart of GX $17+2$ has not yet been unambiguously identified. The difficulties arise mainly because of the position of the source near the Galactic center $\left(l=16^{\circ} .4, b=1.3\right)$, where the chance of superposition to unrelated field objects is very high.

In this paper we report on the results obtained from two BeppoSAX observations of GX 17+2, performed before the October 1999 observation reported by DS2000. In Sect. 2 we describe the observations and data analysis, in Sect. 3 we present the results of the timing and spectral analysis, in Sect. 4 we discuss the results and in Sect. 5 we draw our conclusions.

\section{Observations and data analysis}

We observed the source twice, on 1997 April 3 and 21, with the Narrow Field Instruments (NFIs) on board BeppoSAX (Boella et al. 1997a). The NFIs include a Low-Energy Concentrator Spectrometer (LECS, 0.1-10 keV; Parmar et al. 1997), three Medium-Energy Concentrator Spectrometers (MECS, 1.5-10 keV; Boella et al. 1997b), a High-Pressure Gas Scintillation Proportional Counter (HPGSPC, 4-120 keV; Manzo et al. 1997), and a Phoswich Detection System (PDS, 15-300 keV; Frontera et al. 1997). Both observations were performed with all three MECS units (MECS unit 1 failed on 1997 May 6). Table 1 reports the observations log along with the on-source exposure times of each NFI and the mean $1.8-10 \mathrm{keV}$ and $13-200 \mathrm{keV}$ count rate measured by all the three MECS units and by the four PDS units, respectively. During all pointings the NFIs worked nominally and the source was detected by all of them. Good data were selected from intervals when the NFIs elevation angle was above the Earth limb by at least $5^{\circ}$ and, for the LECS, during the dark earth. The SAXDAS 2.0.0 data analysis package was used for processing the LECS, MECS and HPGSPC data, while the PDS data reduction was performed using the XAS package v2.1 (Chiappetti \& Dal Fiume 1997). The LECS and MECS spectra were extracted from a region of $4^{\prime}$ radius centered on the source position. The background spectrum for the LECS and the MECS was determined using the standard blank field spectra as reported by Fiore et al. (1999), while for the HPGSPC and the PDS the background was estimated with the rocking collimator technique. The spectra of all NFIs were rebinned to reduce the oversampling in the energy resolution and 
to have a minimum of 20 counts in each bin, in order to reliably use the $\chi^{2}$ statistics for spectral fitting. For each instrument we performed the spectral and temporal analysis in the energy range where the response function is well determined; on the basis of this extension, the energy range is $0.4-4.0 \mathrm{keV}$ for LECS, 1.8-10 keV for MECS and 8-30 keV for HPGSPC, while for PDS the range is $13-120 \mathrm{keV}$ in the first observation and 13-40 keV in the second one as the source was not detected beyond $40 \mathrm{keV}$. We performed spectral analysis over four different regions, named I, II, III and IV, according to the $1.8-10 \mathrm{keV}$ MECS count rate being $\lesssim 180 \mathrm{~s}^{-1}$, between $\sim 180$ and $\sim 185 \mathrm{~s}^{-1}$, between $\sim 185$ and $\sim 195 \mathrm{~s}^{-1}$ and $\gtrsim 195 \mathrm{~s}^{-1}$ (see Fig. 1). We note that region I is entirely covered by the points of the first observation while the other regions are covered by the second observation. Only few points of the second observation fall in region I, so we could not extract statistically significant spectral information in this region during the second observation. For the latter we then extracted both the average spectrum and the separated spectra for regions II, III and IV.

We used the XSPEC v11.2.0 package to fit the multiinstrument energy spectra. In the broad-band fits, normalization factors were applied to LECS, HPGSPC and PDS spectra following the cross-calibration tests between them and the MECS, constraining them within the allowed ranges (Fiore et al. 1999). Interstellar photoelectric absorption, modeled using the cross sections implemented in XSPEC and with solar abundances given by Anders \& Ebihara (1982), was included in all spectral models used. Uncertainties in the parameters values obtained from the spectral fits are single parameter errors at $90 \%$ confidence level, while upper limits are reported at $2 \sigma$ level. For the evaluation of the source luminosity we have assumed a distance of $7.5 \mathrm{kpc}$ (Penninx et al. 1988)

\section{Results}

\subsection{Temporal properties}

The source 1.8-10 keV light curve of the two observations is shown in Fig. 1. A flux variability on time scales of hours is apparent and is confirmed by the fit with a constant function, which gives $\chi^{2} /$ d.o.f. $=436 / 104$. Unfortunately, the presence of several gaps in the light curve prevents us to investigate whether the source shows periodic flux modulations. The HID of the two observations, obtained defining as intensity the 1.8-10 keV MECS count rate and as hard colour the countrate ratio in the $6.5-10 \mathrm{keV}$ and $5-6.5 \mathrm{keV}$ energy bands, respectively, is shown in Fig. 2. As it can be seen, the data points of the first observation are all clustered in the left side of the diagram, while those of the second observation, reflecting the source intensity decrease (from $\sim 200 \mathrm{~s}^{-1}$ to $\sim 180 \mathrm{~s}^{-1}$, see Fig. 1, lower panel), trace an extended path (regions II, III and IV) that, on the left side, smoothly merges with the data points of the first observation. However from the shown HID, it is difficult to establish which branch of the Z-track our data correspond to. To solve this issue, we plot in the right panel of Fig. 2 both our data points and those obtained with BeppoSAX by DS2000, when the source clearly traced the $\mathrm{HB}$ and NB. As DS2000 adopted different energy ranges to
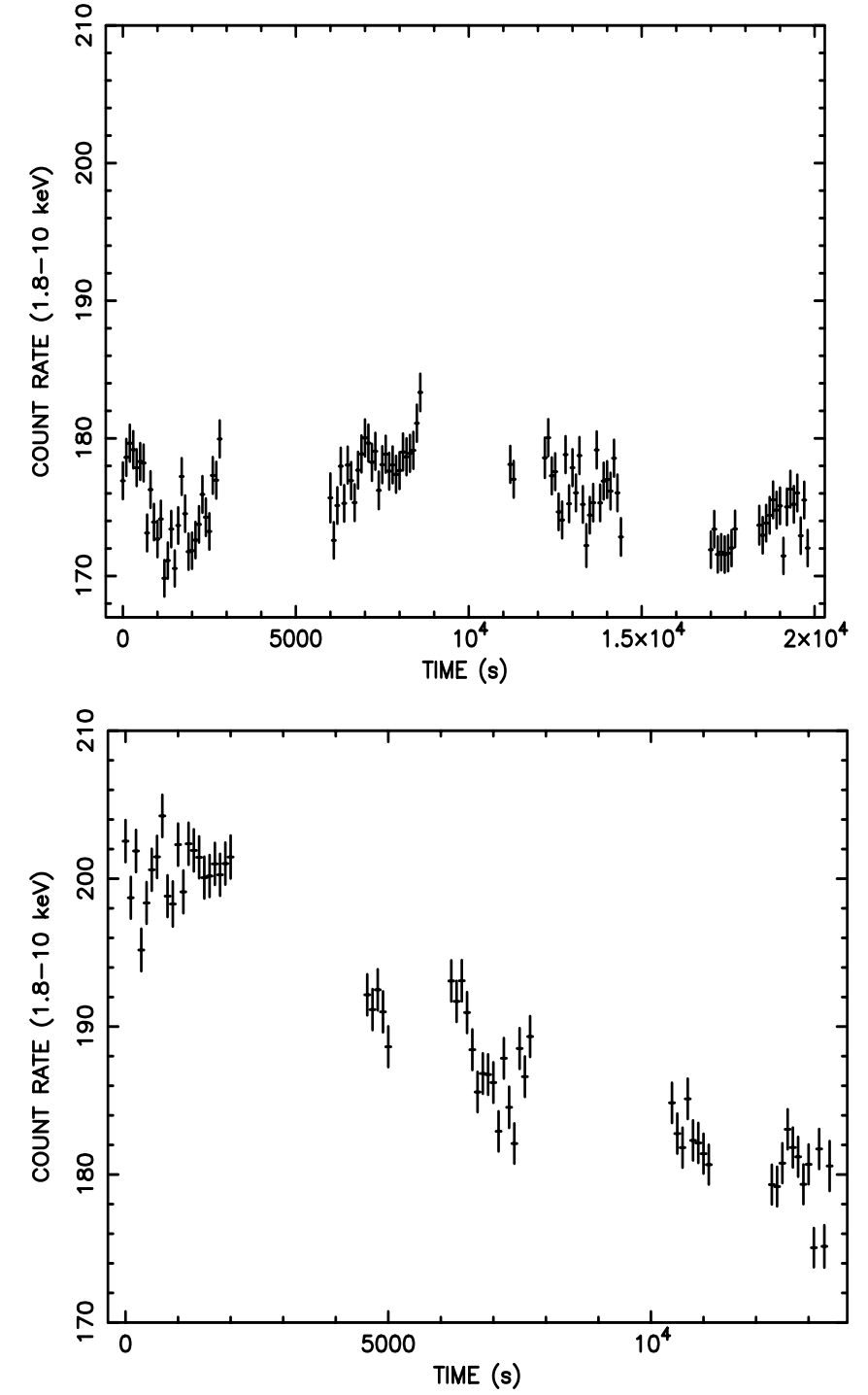

Fig. 1. MECS 1.8-10 keV light curves of the first (upper panel) and second (lower panel) observation of GX $17+2$. The bin size in both cases is $100 \mathrm{~s}$. Time is given in seconds from the beginning of each observation (see Table 1).

produce the HID, we used the MECS event files of their observations from the BeppoSAX public data archive to derive a new HID in the same energy ranges adopted by us. From Fig. 2 it is possible to see that GX $17+2$, during our observations, traced almost the entire HB albeit in a slightly shifted position with respect to that observed by DS2000. Secular shifts of the Z pattern in the HID of GX $17+2$ have been previously observed to occur on time scales of several months by RXTE (Homan et al. 2002) and EXOSAT (Kuulkers et al. 1997). On the others hand, both RXTE and EXOSAT observations revealed strong stability (within $\sim 2 \%$ ) of the hard colour, and this confirms the HB nature of the source during our observations.

We also investigated the source erratic time variability in the $1.8-10 \mathrm{keV}$ energy band (MECS data), by deriving the power spectral density (PSD) in the $8 \times 10^{-4}-50 \mathrm{~Hz}$ frequency range, obtained by averaging data intervals of 131072 points with a binning time of $10^{-2} \mathrm{~s}$, for the first and second 

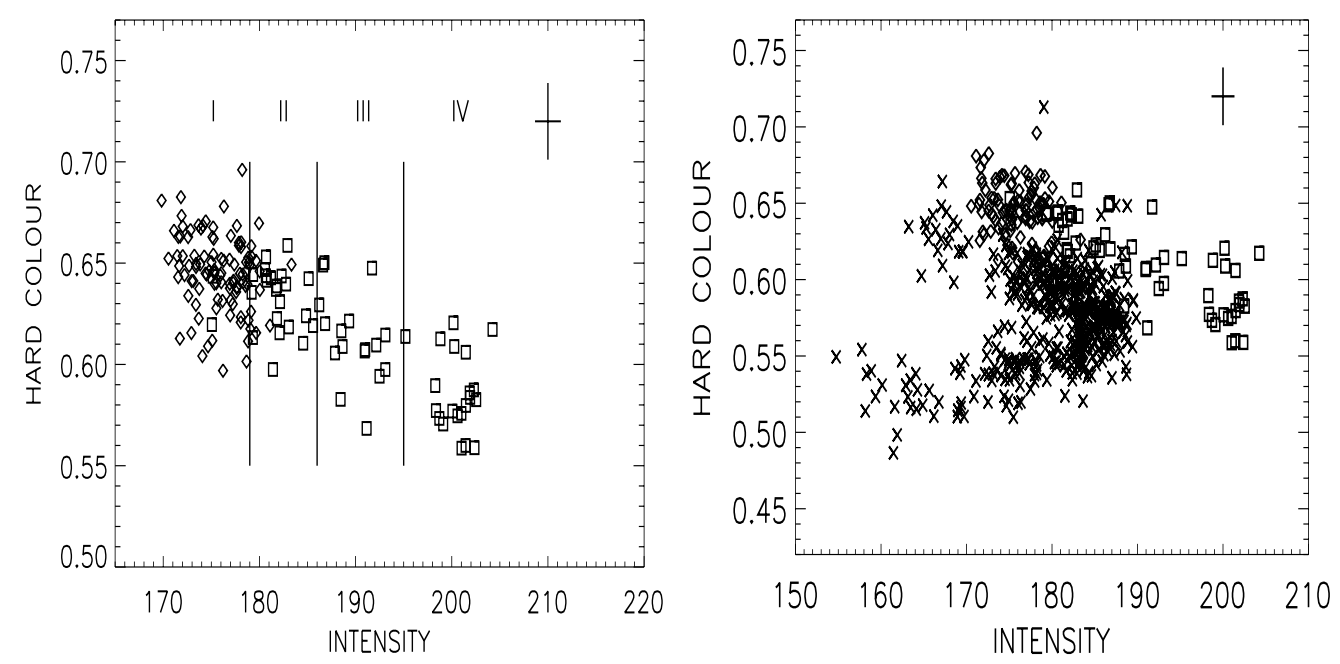

Fig. 2. Left panel: HID of the two BeppoSAX observations of GX 17+2. Diamonds: first observation. Open squares: second observation. The four count-rate regions are also shown. Each point corresponds to a bin size of $100 \mathrm{~s}$. Right panel: source HID of both our observations (same symbols as in the left panel) and that performed by DS2000 (crosses). The bin size of the 1997 October data points is $400 \mathrm{~s}$. A typical error bar is shown on the top right of each panel.
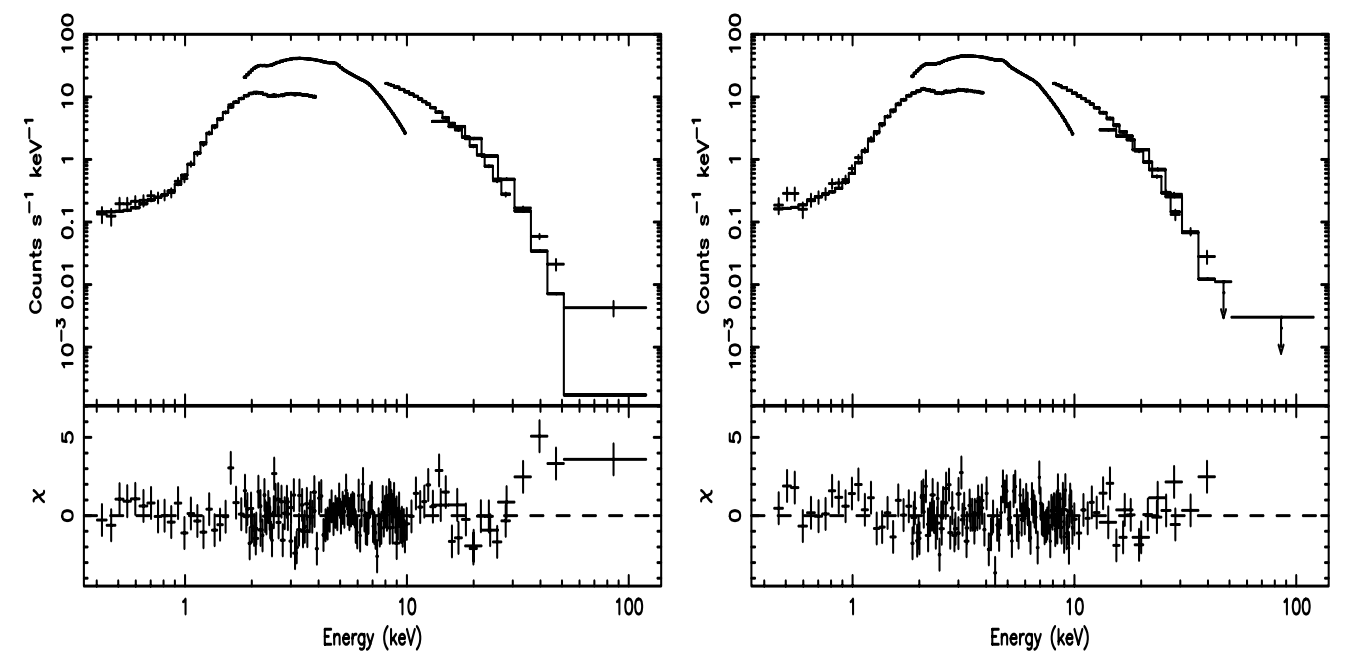

Fig. 3. Count-rate spectra of the first observation (left panel) and of the second observation (right panel) of GX 17+2 and best-fit folded model WABS(BB + COMPTT + Gaussian) with $k T_{\mathrm{bb}}<k T_{\mathrm{s}}$. In the first observation, an absorption edge was also included. Below each panel are shown the residuals to the model in units of $\sigma$. The last two PDS points of the second observation have $S / N<3$, so they were not included in the fit and are reported as $2 \sigma$ upper limits. Similar results are obtained for the same model but with $k T_{\mathrm{bb}}>k T_{\mathrm{s}}$.

observation respectively. Unfortunately, because of the low collecting area of the telescopes, the statistical quality of the derived PSD did not allow us to resolve the broad-band noise components typical of Z sources or to detect QPOs observed with other satellites (EXOSAT, Hasinger \& van der Klis 1990; RXTE, Homan et al. 2002). We thus only derived the integrated root mean square fractional variation of the $1.8-10 \mathrm{keV}$ flux which is $7.1 \% \pm 1.3 \%$ in the first observation and $6.3 \% \pm 2.4 \%$ in the second one.

\subsection{Spectral properties}

Following the results obtained on GX $17+2$ by DS2000, we first attempted to fit the source spectra with the BB + COMPTT continuum model plus a fluorescence $\mathrm{Fe}$ line and an absorption Fe K-edge. The best-fit results are shown in Fig. 3, where the time-averaged count-rate spectra of the observations 1 and 2 are shown along with the residuals to this model. As can be seen, for the second observation (see Fig. 3, right panel) the model describes quite well the data, with no apparent systematic residuals, even though the $\chi^{2}$ is not satisfactory $\left(\chi^{2} /\right.$ d.o.f. $\left.=192 / 141\right)$. This could be due to some spectral evolution of the source with time (see Sect. 3.3.2). In addition, no significant K-edge is required by the data (see Table 2). Unlike the second observation, the above model does not provide a good description of the data of the first observation, with a $\chi^{2} /$ d.o.f. $=222 / 144$, mainly due to the residuals to the bestfit model above $30 \mathrm{keV}$ (see Fig. 3, left panel). It is sufficient to add, as also done by DS2000, a PL component to the above spectral model to get a very good fit $\left(\chi^{2} /\right.$ d.o.f. $\left.=143 / 142\right)$. The best-fit results are shown in Table 2 . A feature of the data obtained from both observations when they are fit with the above 
Table 2. Best-fit parameters of the multi-component model WABS(BB + COMPTT + Gaussian + K-edge + PL) for the first and second observation of GX 17+2. The two solutions of the model are reported. The F-test gives the probability of chance improvement of the $\chi^{2}$ for the addition of the PL. The ratios $L_{\mathrm{bb}} / L_{\mathrm{tot}}$ and $L_{\mathrm{pl}} / L_{\mathrm{tot}}$ are computed in the $0.4-200 \mathrm{keV}$ energy range.

\begin{tabular}{|c|c|c|c|c|}
\hline & $\begin{array}{c}\text { Obs. } 1 \\
\text { I }\end{array}$ & $\begin{array}{c}\text { Obs. } 2 \\
\text { II+III+IV }\end{array}$ & $\begin{array}{c}\text { Obs. } 1 \\
\text { I }\end{array}$ & $\begin{array}{c}\text { Obs. } 2 \\
\text { II+III+IV }\end{array}$ \\
\hline Parameter & \multicolumn{2}{|c|}{ Solution 1} & \multicolumn{2}{|c|}{ Solution 2} \\
\hline$N_{\mathrm{H}}^{\mathrm{a}}$ & $2.5_{-0.3}^{+0.2}$ & $2.2_{-0.2}^{+0.1}$ & $2.5_{-0.3}^{+0.2}$ & $2.3_{-0.1}^{+0.1}$ \\
\hline$k T_{\mathrm{bb}}(\mathrm{keV})$ & $0.66_{-0.03}^{+0.04}$ & $0.61_{-0.03}^{+0.03}$ & $1.79_{-0.16}^{+0.10}$ & $1.38_{-0.02}^{+0.05}$ \\
\hline$R_{\mathrm{bb}}(\mathrm{km})$ & $35_{-4}^{+4}$ & $40_{-4}^{+4}$ & $3.7_{-0.6}^{+0.6}$ & $7.4_{-0.5}^{+0.5}$ \\
\hline$k T_{\mathrm{s}}(\mathrm{keV})$ & $1.24_{-0.08}^{+0.09}$ & $1.09_{-0.04}^{+0.06}$ & $0.58_{-0.02}^{+0.02}$ & $0.55_{-0.03}^{+0.01}$ \\
\hline$k T_{\mathrm{e}}(\mathrm{keV})$ & $3.4_{-0.1}^{+0.1}$ & $3.3_{-0.1}^{+0.1}$ & $3.5_{-0.1}^{+0.1}$ & $3.15_{-0.04}^{+0.06}$ \\
\hline$\tau$ & $11.0_{-0.5}^{+0.4}$ & $10.5_{-0.4}^{+0.4}$ & $12.0_{-0.5}^{+0.6}$ & $13.0_{-0.5}^{+0.3}$ \\
\hline$\Gamma$ & $2.8_{-0.2}^{+0.1}$ & {$[2.8]$} & $2.8_{-0.2}^{+0.1}$ & {$[2.8]$} \\
\hline$N_{\mathrm{pl}}^{\mathrm{b}}$ & $2.1_{-1.2}^{+1.2}$ & $0.7_{-0.6}^{+0.6}$ & $2.3_{-1.3}^{+1.2}$ & $1.1_{-0.6}^{+0.2}$ \\
\hline F-test & $3 \times 10^{-14}$ & 0.11 & $5 \times 10^{-12}$ & 0.03 \\
\hline$E_{1}(\mathrm{keV})$ & $6.7_{-0.1}^{+0.1}$ & $6.7_{-0.1}^{+0.1}$ & $6.7_{-0.1}^{+0.1}$ & $6.7_{-0.1}^{+0.1}$ \\
\hline$\sigma_{1}(\mathrm{keV})$ & $0.1_{-0.1}^{+0.1}$ & $0.2_{-0.1}^{+0.1}$ & $0.1_{-0.1}^{+0.1}$ & $0.2_{-0.1}^{+0.1}$ \\
\hline$I_{1}^{c}$ & $3.6_{-0.8}^{+1.2}$ & $4.6_{-1.4}^{+1.2}$ & $3.8_{-0.8}^{+1.2}$ & $4.5_{-1.0}^{+1.6}$ \\
\hline$E W_{1}(\mathrm{eV})$ & $26_{-6}^{+9}$ & $32_{-9}^{+9}$ & $27_{-6}^{+8}$ & $31_{-7}^{+11}$ \\
\hline$E_{\text {edge }}(\mathrm{keV})$ & $8.2_{-0.3}^{+0.4}$ & [8.2] & $8.3_{-0.2}^{+0.3}$ & [8.3] \\
\hline$\tau_{\text {edge }}\left(10^{-2}\right)$ & $2.5_{-0.7}^{+1.4}$ & $<2.0$ & $3.3_{-1.5}^{+1.1}$ & $<1.8$ \\
\hline$L_{\mathrm{bb}} / L_{\mathrm{tot}}$ & 0.16 & 0.17 & 0.10 & 0.15 \\
\hline$L_{\mathrm{pl}} / L_{\mathrm{tot}}$ & 0.30 & 0.13 & 0.32 & 0.18 \\
\hline$L_{0.4-30 \mathrm{keV}}^{\mathrm{d}}$ & 1.87 & 1.62 & 1.91 & 1.69 \\
\hline$L_{30-200 \mathrm{keV}}^{\mathrm{d}}$ & 0.02 & 0.01 & 0.02 & 0.01 \\
\hline$\chi^{2} /$ d.o.f. & $143 / 142$ & $188 / 140$ & $140 / 142$ & $189 / 140$ \\
\hline
\end{tabular}

${ }^{\mathrm{a}}$ In units of $10^{22} \mathrm{~cm}^{-2}$.

${ }^{\mathrm{b}}$ Photons $\mathrm{keV}^{-1} \mathrm{~cm}^{-2} \mathrm{~s}^{-1}$ at $1 \mathrm{keV}$.

${ }^{c}$ Total photons in the line in units of $10^{-3} \mathrm{~cm}^{-2} \mathrm{~s}^{-1}$.

${ }^{\mathrm{d}}$ Unabsorbed luminosity in units of $10^{38} \mathrm{erg} \mathrm{s}^{-1}$.

model (with or without the PL) is that two equally acceptable solutions for the values of the BB temperature $k T_{\mathrm{bb}}$ and temperature of the seed photons $k T_{\mathrm{s}}$ are found (see Table 2): one with $k T_{\mathrm{bb}}<k T_{\mathrm{s}}$ and the other with $k T_{\mathrm{bb}}>k T_{\mathrm{s}}$, while the other parameter values remain substantially unchanged within errors.

Finally, to test the physical hypothesis of the threelayered atmospheric structure in accretion disks suggested by Zhang et al. (2000) for the black hole candidates (BHC) GRO J1655-40 and GRS 1915+105, in the first observation we replaced the PL component with a further COMPTT model with higher electron temperature, which we label as $k T_{\mathrm{e}}^{h}$. Approximating the seed photons temperature of this hotter electrons to the temperature of the electrons of the underlying warm layer (an assumption justified by the fact that the Compton parameter $y$ of the warm electrons is $\sim 3$, so that their emerging spectrum is not far from a Wien-like), namely keeping $k T_{\mathrm{s}}^{h}=k T_{\mathrm{e}}$, does not give any stable solution. A good fit is obtained by keeping free all the model parameters $\left(\chi^{2} /\right.$ d.o.f. $\left.=149 / 140\right)$, but in this case, unlike the results by Zhang et al. (2000), the seed photons temperature of the hotter COMPTT component $\left(k T_{\mathrm{s}}^{h} \sim 1.3 \mathrm{keV}\right.$, with a corresponding $k T_{\mathrm{e}}^{h} \sim 75 \mathrm{keV}$ and $\left.\tau^{h} \sim 0.05\right)$ is quite different from the temperature $(\sim 8 \mathrm{keV})$ of the warm electrons.

\subsection{A physical model for the hard X-ray tail}

In order to investigate the physical origin of the hard X-ray tail detected during the first observation of GX $17+2$, we used the Comptonization model worked out by Coppi (1999, EQPAIR in XSPEC), which has the advantage of being general and can be used to fit the source spectra of both observations. In this model, non-thermal electrons with energy spectrum $\propto \gamma^{-p}$ (where $\gamma$ is the Lorentz factor) are injected in the plasma cloud at a rate $\propto \gamma^{-p}$. These electrons lose energy because of Compton, Coulomb and bremsstrahlung processes and thus establish a steady-state distribution. At high energies (for $\gamma>\gamma_{\min }$ up to a certain value of $\gamma_{\max }$ ) the distribution is non-thermal (power-law like), but at low energies it joins the Maxwellian distribution and the final result of this process is a hybrid thermal plus non-thermal plasma. The temperature 

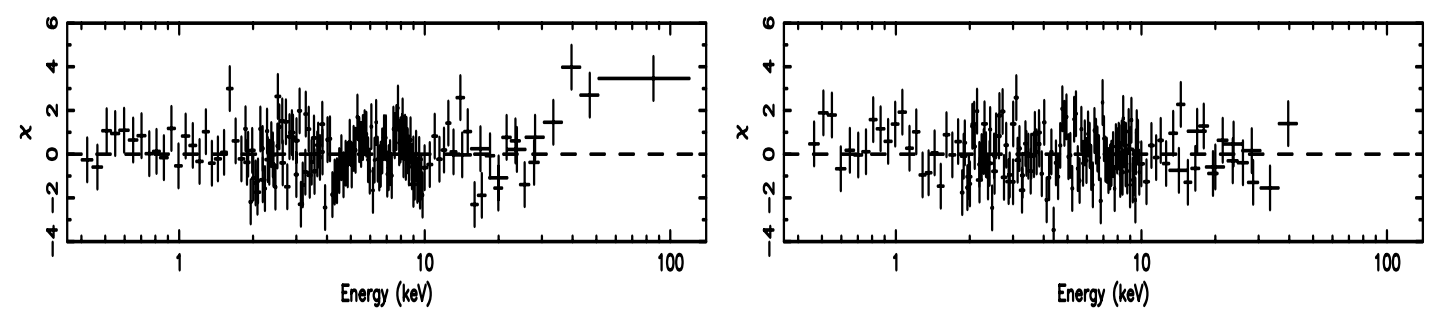

Fig. 4. Residuals in units of $\sigma$ to the model WABS(BB + EQPAIR + Gaussian), assuming purely thermal Comptonization, for the first (left panel) and for second (right panel) observation of GX 17+2.

of the thermal distribution, $k T_{\mathrm{e}}$, is self-consistently computed by means of energy balance and is not a free parameter of the model. The plasma properties are characterized by the powers $L$ supplied to the different components. A compactness parameter is introduced and defined as:

$\ell \equiv \frac{\sigma_{\mathrm{T}}}{m_{\mathrm{e}} c^{2}} \frac{L}{R}$

where $R$ is the characteristic size of the region and $\sigma_{\mathrm{T}}$ is the Thomson cross-section for scattering. The compactness of the input soft photons is parametrized by $\ell_{\mathrm{s}}$. The power supplied to the Comptonizing electrons is parametrized as $\ell_{\mathrm{h}}=\ell_{\mathrm{th}}+\ell_{\mathrm{nth}}$, where $\ell_{\text {th }}$ is the power supplied to heat thermal particles, while $\ell_{\text {nth }}$ is the power going into accelerating non-thermal particles. If $\ell_{\text {nth }}=0$, then one has a purely thermal (Maxwellian) distribution. EQPAIR can also test the presence of Compton reflection of the primary photon spectrum from a neutral or a ionized medium (Magdziarz \& Zdziarski 1995), allowing for different metal abundances with respect to the solar ones.

\subsubsection{First observation}

We first assumed that all the power goes in heating the thermal particles $\left(\ell_{\mathrm{nth}} / \ell_{\mathrm{h}}=0\right.$, purely thermal Comptonization) with a simple BB spectrum of the seed photons. We added to EQPAIR also a direct $\mathrm{BB}$ component plus a Fe $\mathrm{K}$ fluorescence line in the source model, as discussed in Sect. 3.2. This model well describes the data below $30 \mathrm{keV}$ but, as in the case of COMPTT, a systematic excess is again observed in the residuals above this threshold (see Fig. 4, left panel). We thus left free to vary the $\ell_{\text {nth }}$ parameter: since the statistics of the data do not allow us to simultaneously constrain $\gamma_{\min }, \gamma_{\max }$ and $p$ in the fits, $\gamma_{\max }$ was frozen at value of 12 (the $\chi^{2}$ is poorly sensitive to $\gamma_{\max }$ in the range from 2 to 50). The chosen value of $\gamma_{\max }$ takes into account the energy range in which the excess over thermal Comptonization is located and the temperature of the seed photons found from the fit. As can be seen from Fig. 5 (left panel), the agreement between the model and the data is excellent. The probability given by the F-test that the $\chi^{2}$ reduction obtained from the inclusion of a non-thermal component in the Comptonizing cloud is due to chance is very low (see Table 3). The presence of a Compton reflection component, assuming solar abundances and a low inclination angle $i=30^{\circ}$ (GX 17+2 is a Sco-like source, Kuulkers \& van der Klis 1996), is only marginally detected $\left(\Delta \chi^{2} \sim 7\right.$, probability of chance improvement of $\sim 3 \%$ ) with best-fit value of the covering factor $\Omega / 2 \pi \sim 0.04$. The ionization parameter value we find is
Table 3. Best-fit parameters of the multi-component model WABS(BB + EQPAIR + Gaussian) for the first and second observation of GX 17+2. The F-test gives the probability of chance improvement of the $\chi^{2}$ switching from thermal to hybrid Comptonization. The compactness parameters $\ell_{\mathrm{s}}, \ell_{\mathrm{h}}$ and $\ell_{\mathrm{nth}}$ are defined in Sect. 3.3. The ratio $L_{\mathrm{bb}} / L_{\mathrm{tot}}$ is computed in the energy range $0.4-200 \mathrm{keV}$.

\begin{tabular}{ccc}
\hline \hline Parameter & Obs. 1 & Obs. 2 \\
& $\mathrm{I}$ & $\mathrm{II}+\mathrm{III}+\mathrm{IV}$ \\
\hline$N_{\mathrm{H}}^{\mathrm{a}}$ & $2.00_{-0.05}^{+0.05}$ & $1.97_{-0.06}^{+0.07}$ \\
$k T_{\mathrm{bb}}(\mathrm{keV})$ & $0.58_{-0.01}^{+0.02}$ & $0.60_{-0.02}^{+0.01}$ \\
$R_{\mathrm{bb}}(\mathrm{km})$ & $33_{-2}^{+2}$ & $31_{-3}^{+3}$ \\
$k T_{\mathrm{s}}(\mathrm{keV})$ & $0.88_{-0.08}^{+0.14}$ & $0.95_{-0.05}^{+0.03}$ \\
$k T_{\mathrm{e}}(\mathrm{keV})$ & 3.5 & 3.5 \\
$\ell_{\mathrm{s}}$ & $7600_{-3400}^{+7000}$ & {$[7600]$} \\
$\ell_{\mathrm{h}} / \ell_{\mathrm{s}}$ & $0.87_{-0.02}^{+0.02}$ & $0.63_{-0.03}^{+0.01}$ \\
$\ell_{\mathrm{nth}} / \ell_{\mathrm{h}}$ & $0.11_{-0.02}^{+0.02}$ & $<0.04$ \\
$\tau$ & $8.4_{-0.7}^{+1.5}$ & $8.4_{-0.2}^{+0.1}$ \\
$p$ & $1.7_{-0.6}^{+1.1}$ & {$[1.7]$} \\
$\gamma_{\mathrm{min}}$ & $1.15_{-0.15}^{+1.54}$ & {$[1.15]$} \\
$\gamma_{\mathrm{max}}$ & {$[12]$} & {$[12]$} \\
$\mathrm{F}-\mathrm{test}$ & $4 \times 10^{-11}$ & 0.07 \\
\hline$E_{\mathrm{l}}(\mathrm{keV})$ & $6.7_{-0.1}^{+0.1}$ & $6.7_{-0.1}^{+0.1}$ \\
$\sigma_{\mathrm{l}}(\mathrm{keV})$ & $0.2_{-0.1}^{+0.1}$ & $0.2_{-0.1}^{+0.1}$ \\
$I_{1}^{b}$ & $4.4_{-0.8}^{+1.3}$ & $4.8_{-0.8}^{+2.2}$ \\
$E W_{\mathrm{l}}(\mathrm{eV})$ & $32_{-6}^{+9}$ & $33_{-6}^{+15}$ \\
\hline$L_{\mathrm{bb}} / L_{\mathrm{tot}}$ & 0.11 & 0.11 \\
$L_{0.4-30 \mathrm{keV}}^{\mathrm{c}}$ & 1.44 & 1.46 \\
$L_{30-200 \mathrm{keV}}^{\mathrm{c}}$ & 0.019 & 0.006 \\
$\chi^{2} / \mathrm{d} . \mathrm{o.f}$. & $143 / 142$ & $185 / 141$ \\
\hline 2
\end{tabular}

${ }^{\mathrm{a}}$ In units of $10^{22} \mathrm{~cm}^{-2}$.

b Total photons in the line in units of $10^{-3} \mathrm{~cm}^{-2} \mathrm{~s}^{-1}$. ${ }^{\mathrm{c}}$ Unabsorbed luminosity in units of $10^{38} \mathrm{erg} \mathrm{s}^{-1}$.

$\xi \sim 800 \mathrm{erg} \mathrm{cm} \mathrm{s}^{-1}$. An important issue to point out is that, unlike COMPTT, with EQPAIR there is not a double solution as concerns the direct $\mathrm{BB}$ and seed photons temperature values.

Replacing the direct BB component with a DBB, we still obtain an acceptable, albeit a bit worse, result $\left(\chi^{2} /\right.$ d.o.f. $=$ $156 / 142)$. If, on the other hand, we assume a DBB seed photon spectrum in EQPAIR plus a direct BB model, the fit is even worse $\left(\chi^{2} /\right.$ d.o.f. $\left.=163 / 142\right)$. 

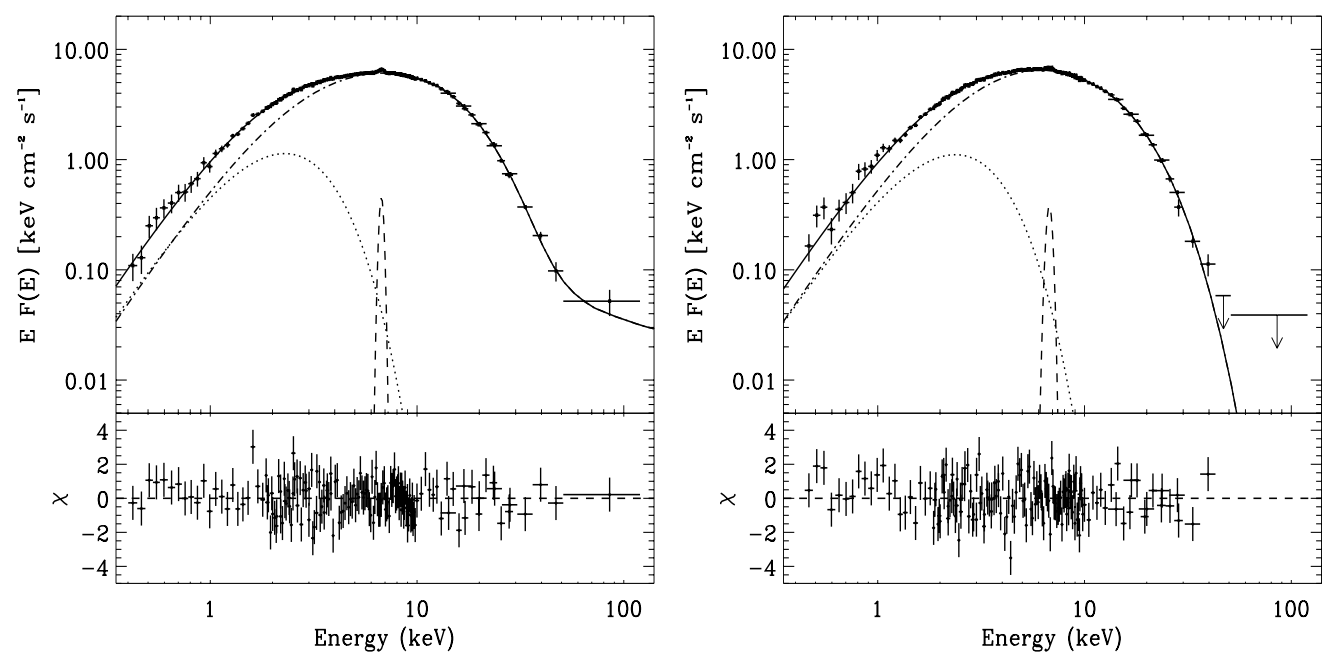

Fig. 5. Unabsorbed $E F(E)$ spectrum of the first (left panel) and of the second (right panel) observation of GX 17+2 along with the best-fit model WABS(BB + EQPAIR + Gaussian). A hybrid spectrum of the Comptonizing electrons is assumed. The $2 \sigma$ upper limit of the last two PDS points of the second observation is also reported. Below each panel, the residuals between the data and the model in units of $\sigma$ are shown. Different line styles represent the single components. Dotted: BB. Dotted-dashed: EQPAIR. Dashed: Gaussian emission line.

\subsubsection{Second observation}

As reported in Sect. 3.2, during the second observation there was slight evidence of a spectral evolution of the source, suggested by the somewhat worse fit of the COMPTT model to the data, in spite of the fact that no systematic residuals to the best-fit model are apparent (see Fig. 3, right panel). This result is confirmed also by using the EQPAIR model, assuming purely thermal Comptonization, to fit the whole observation $\left(\chi^{2} /\right.$ d.o.f. $=186 / 142$, see Fig. 4 , right panel). In this case we also find that the soft compactness $\ell_{\mathrm{s}}$ is unconstrained so, during the fit, we fixed it at the best-fit value of the first observation. In order to put an upper limit on the power supplied in accelerating non-thermal electrons, we allowed the $\ell_{\mathrm{nth}} / \ell_{\mathrm{h}}$ parameter to vary, by keeping $\gamma_{\min }$ and $p$ frozen at the best-fit values of the first observation. The results are reported in Table 3. As it can be seen, the power going in accelerating non-thermal electrons is consistent with zero and its $2 \sigma$ upper limit is lower than the value of the first observation. It is worth pointing out that this upper limit value on $\ell_{\mathrm{nth}} / \ell_{\mathrm{h}}$ is obtained even including in the fit the last two PDS points (see Fig. 3, right panel), in order to have the same energy coverage of the first observation. The $2 \sigma$ upper limit on the 40-120 keV source flux, assuming as best-fit model the one reported in Table 3, is $\sim 7 \times 10^{-11} \mathrm{erg} \mathrm{cm}^{-2} \mathrm{~s}^{-1}$ (see Fig. 5, right panel) a value below that found in the first observation $\left(\sim 1.0 \times 10^{-10} \mathrm{erg} \mathrm{cm}^{-2} \mathrm{~s}^{-1}\right)$. We subsequently performed spectral analysis separately for the three regions of the HID labeled in Fig. 2; the results are reported in Table 4.

\section{Discussion}

Some relevant results have been derived from our observations of GX 17+2 with BeppoSAX. The persistent continuum X-ray emission of GX $17+2$ can be described by several twocomponent models. When we adopt the COMPTT model plus a BB, we find that two solutions are possible, which are reminiscent of the well known Eastern (Mitsuda et al. 1984, 1989)
Table 4. Same as in Table 3 but for regions II, III and IV of the second observation as reported in Fig. 2 and assuming thermal Comptonization.

\begin{tabular}{cccc}
\hline \hline Parameter & II & III & IV \\
\hline$N_{\mathrm{H}}^{\mathrm{a}}$ & $2.03_{-0.05}^{+0.11}$ & $2.05_{-0.13}^{+0.10}$ & $1.93_{-0.14}^{+0.18}$ \\
$k T_{\mathrm{bb}}(\mathrm{keV})$ & $0.61_{-0.03}^{+0.01}$ & $0.53_{-0.06}^{+0.09}$ & $0.55_{-0.06}^{+0.08}$ \\
$R_{\mathrm{bb}}(\mathrm{km})$ & $37_{-3}^{+3}$ & $32_{-10}^{+10}$ & $29_{-9}^{+9}$ \\
$k T_{\mathrm{s}}(\mathrm{keV})$ & $1.17_{-0.07}^{+0.02}$ & $0.85_{-0.08}^{+0.05}$ & $0.90_{-0.04}^{+0.10}$ \\
$k T_{\mathrm{e}}(\mathrm{keV})$ & 4.0 & 3.5 & 3.1 \\
$\ell_{\mathrm{s}}$ & {$[7600]$} & {$[7600]$} & {$[7600]$} \\
$\ell_{\mathrm{h}} / \ell_{\mathrm{s}}$ & $0.47_{-0.03}^{+0.01}$ & $0.71_{-0.03}^{+0.04}$ & $0.59_{-0.02}^{+0.02}$ \\
$\tau$ & $6.7_{-0.5}^{+0.2}$ & $8.6_{-0.3}^{+0.4}$ & $8.9_{-0.2}^{+0.3}$ \\
\hline$E_{\mathrm{l}}(\mathrm{keV})$ & $6.6_{-0.1}^{+0.1}$ & $6.7_{-0.2}^{+0.2}$ & $6.8_{-0.1}^{+0.1}$ \\
$\sigma_{\mathrm{l}}(\mathrm{keV})$ & $0.2_{-0.2}^{+0.4}$ & $0.3_{-0.2}^{+0.3}$ & $0.1_{-0.1}^{+0.2}$ \\
$I_{1}^{b}$ & $5.0_{-2.3}^{+3.6}$ & $6.5_{-3.1}^{+4.8}$ & $5.3_{-1.8}^{+2.4}$ \\
$E W_{1}(\mathrm{eV})$ & $35_{-16}^{+25}$ & $44_{-21}^{+33}$ & $36_{-12}^{+16}$ \\
\hline$L_{\mathrm{bb}} / L_{\mathrm{tot}}$ & 0.17 & 0.07 & 0.07 \\
$L_{0.4-30 \mathrm{keV}}^{\mathrm{c}}$ & 1.47 & 1.50 & 1.48 \\
$L_{30-200 \mathrm{keV}}^{\mathrm{c}}$ & 0.01 & 0.007 & 0.004 \\
$\chi^{2} / \mathrm{d} . \mathrm{o.f}$. & $145 / 142$ & $133 / 142$ & $145 / 142$ \\
\hline
\end{tabular}

a In units of $10^{22} \mathrm{~cm}^{-2}$.

${ }^{b}$ Total photons in the line in units of $10^{-3} \mathrm{~cm}^{-2} \mathrm{~s}^{-1}$.

${ }^{\mathrm{c}}$ Unabsorbed luminosity in units of $10^{38} \mathrm{erg} \mathrm{s}^{-1}$.

and Western (White et al. 1986, 1988) models. Indeed, looking at the results of Table 2, for solution 1, we can interpret the $\sim 0.6 \mathrm{keV}$ BB as the direct emission from the accretion disk, with Comptonization mainly occurring in the hotter boundary layer $\left(k T_{\mathrm{s}} \sim 1.2 \mathrm{keV}\right.$, Eastern Model scenario). But the same spectrum can be described (solution 2) by a $\sim 1.5 \mathrm{keV}$ BB plus a Comptonization region of cooler photons $\left(k T_{\mathrm{s}} \sim 0.6 \mathrm{keV}\right)$. 
In this case the direct BB emission can be interpreted as coming from the boundary layer, while Comptonization takes place in the disk (Western model scenario). From a statistical point of view, we do not find strong reasons for favouring one solution over the other; however, the former solution is the only one we find when COMPTT is replaced by EQPAIR.

The soft compactness estimated by EQPAIR (see Table 3) is much higher than that reported in the case of BHCs (e.g., Frontera et al. 2001; Gierliński \& Done 2003); this is however not surprising given that in neutron star systems, unlike $\mathrm{BHCs}$, the source environment in the inner disk region has an additional source of soft luminosity coming from the neutron star. Apart from this crucial difference, related to the nature of the compact object, there are some physical characteristics which link in some way GX $17+2$ to BHCs. First, the spectral index of the non-thermal injected electrons $(p \sim 1.7)$ is consistent, within errors, with that found, e.g., in Cyg X-1 (Gierliński et al. 1999; Frontera et al. 2001) and GRS 1915+105 (Zdziarski et al. 2001). Moreover, the spectral shape of the source in the $E F(E)$ diagram (see Fig. 5) strongly resembles that of BHCs in the so-called ultrasoft state, when $L_{X} \sim L_{\text {Edd }}$ (e.g., GX 339-4, GRS 1915+105, XTE J1550-564, see the review by Zdziarski \& Gierliński 2004), where the peak of the energy emission occurs below $10 \mathrm{keV}$. The similarity with GRS 1915+105, in particular, results even more intriguing looking at the X-ray spectral parameters found by Zdziarski et al. (2001) by fitting the source RXTE/OSSE 3-600 keV X-ray spectrum with EQPAIR: the authors indeed found $k T_{\mathrm{e}} \sim 3.6 \mathrm{keV}, \tau \sim 4.4$, $k T_{\mathrm{s}} \sim 1.3 \mathrm{keV}$ and $\ell_{\text {nth }} / \ell_{\mathrm{th}} \sim 0.1$. Only the ratio $\ell_{\mathrm{h}} / \ell_{\mathrm{s}}$ was lower $(\sim 0.3)$ than that found in GX $17+2$ by us. As $\mathrm{Z}$ sources stably accrete at near-Eddington rate, these similarities lead to suggest that Comptonization/acceleration processes in X-ray binary systems in this phase do not strongly depend on the presence or not of a solid surface at the inner boundary of the accretion disk.

An important result of our study concerns the transient high-energy X-ray tail. We confirm its presence when the source is in the upper left $\mathrm{HB}$ of the $\mathrm{Z}$ diagram, as already observed by DS2000. But, unlike DS2000, who found that the hard X-ray excess gradually decreased as the source moved towards right in the $\mathrm{HB}$, completely disappearing when the source achieved the NB, we find that this disappearance occurs when the source is still in the HB, in particular during its transition from region I to region II of the HID (Fig. 2). The hard X-ray excess is described by a PL with the same photon index found by DS2000 ( $\Gamma \sim 2.8$, see Table 2$)$. In order to gain some insight on the physical origin of the hard X-ray component, we replaced the COMPTT model with the Comptonization model EQPAIR of Coppi (1999). In this model, in addition to a thermal population of electrons, a non-thermal electron tail can be included. Assuming a purely Maxwellian distribution of the electron energies, the hard X-ray excess is still apparent above the BB plus EQPAIR model (see Fig. 4). Only assuming a thermal plus non-thermal plasma the EQPAIR model gives a good description of the high-energy excess. To our knowledge, this is the first time that the transient hard X-ray tail of a Z source (see Sect. 1) is described in terms of hybrid Comptonization.
An important question is whether the two electron populations (thermal and non-thermal) are physically connected. Unfortunately the present data do not allow to answer this question. We consider two cases separately. If the two electron populations are not physically related, a likely origin of the nonthermal electrons is a relativistic jet escaping the system. In recent years, relativistic jets have revealed to be a quite common property of LMXBs, providing a link between these systems and AGNs (for a review see, e.g., Fender 2002). In this thermal plus non-thermal scenario, the Comptonized spectrum is produced by a thermal corona (likely located above the inner disk close to the boundary layer) plus a relativistic nonthermal electron jet, with the soft seed photons mainly coming from the inner disk. The energy of the seed photons $(\sim 1 \mathrm{keV}$, see Table 3 ) is indeed consistent with the value expected for the inner disk temperature of LMXBs hosting a neutron star (see, e.g., the review by Barret 2001). Thus we could tentatively identify the decrease of the power supplied to non-thermal electrons $\left(\ell_{\mathrm{nth}} / \ell_{\mathrm{h}}\right.$ drops from $\sim 0.1$ to less than $\sim 0.04$ from the first to the second observation), as a switching-off of the mechanism producing the jet.

On the other hand, if the two electron populations are physically related, a possible origin of the non-thermal component is in processes of magnetohydrodynamic turbulence occurring in the corona (e.g., magnetic reconnection), in analogy with what observed in the solar corona (Crosby et al. 1998; see also the review by Coppi 1999). In this case particle acceleration cannot be $100 \%$ efficient in the production of non-thermal high-energy particles (e.g., Zdziarski et al. 1993). This can occur only above a certain momentum threshold when the acceleration time scale for a particle becomes significantly shorter than its corresponding thermalization (or energy-exchange) time scale. Below this threshold particles are tightly coupled and their acceleration goes mainly in a bulk heating of the thermal plasma component.

We have also explored the physical scenario proposed by Zhang et al. (2000) for GRO J1655-40 and GRS 1915+105, in which a much hotter, optically thin thermal corona is located above a warm layer with lower temperature and high opacity. This scenario seems not suitable for GX $17+2$. In fact, in this hypothesis, one would expect that the seed photons of the hotter layer are those coming from the underlying one, and thus should have a temperature similar to that of the colder electrons $\left(k T_{\mathrm{e}}^{w} \sim 3-5 \mathrm{keV}\right)$. This is indeed what Zhang et al. (2000) found with $A S C A / R X T E$ data in the case of GRO J1655-40 and GRS $1915+105$. However, in our case $k T_{\mathrm{s}}^{h}$ significantly differs from $k T_{\mathrm{e}}^{w}(\sim 1.3 \mathrm{keV}$ and $\sim 8 \mathrm{keV}$, respectively), thus ruling out the three-layered accretion hypothesis.

In addition to the origin of the hard X-ray emission, its correlation with the position of $\mathrm{Z}$ sources in the $\mathrm{CD} / \mathrm{HID}$ is still unclear. As discussed in Sect. 1, the results obtained from GX 17+2, Cyg X-2 and GX 5-1 (but not, however, from Sco X-1), could suggest the possibility of an anti-correlation between presence of hard X-ray emission and accretion rate $\dot{M}$ in $\mathrm{Z}$ sources, in a similar fashion to what observed both in atoll sources and BHCs, where the hard spectra are usually observed during the low inferred $\dot{M}$ states (e.g., Barret \& Olive 2002; McClintock \& Remillard 2004). Our results on GX 17+2 
do not contrast with this possibility but, when compared with those found by DS2000, they point to the presence of a threshold, that we thus tentatively identify with a threshold in $\dot{M}$, above which the hard tail disappears. A definite answer to this suggestion could come from very sensitive monitoring of the source at high energies in region I of Fig. 2 during the second observation, but this was not possible because of the too few points falling in that region.

An important issue is what we mean by $\dot{M}$ : it appears evident that this $\dot{M}$ is not the one that drives the X-ray luminosity, since the latter does not change along the track (see Tables 3 and 4). Thus, if some $\dot{M}$ increases along the Z-track, it is not correlated with the X-ray luminosity: an intriguing way out to this problem was proposed by van der Klis (2001) to explain the "parallel tracks" phenomenon. He suggested that what does change along the track is not the prompt disk accretion rate $\dot{M}_{\mathrm{d}}(t)$ but rather the ratio $\eta=\dot{M}_{\mathrm{d}}(t) /\left\langle\dot{M}_{\mathrm{d}}(t)\right\rangle$, where $\left\langle\dot{M}_{\mathrm{d}}(t)\right\rangle$ is the long-term $\dot{M}_{\mathrm{d}}(t)$ average. The luminosity is supposed to be related to the sum of $\dot{M}_{\mathrm{d}}(t)$ plus $\left\langle\dot{M}_{\mathrm{d}}(t)\right\rangle$ $\left(L_{\mathrm{X}} \propto \dot{M}_{\mathrm{d}}(t)+\alpha\left\langle\dot{M}_{\mathrm{d}}(t)\right\rangle\right)$ while the curve length $\mathrm{S}$ across the track is a measure of $\eta$. The threshold above which we see the disappearance of the hard tail could thus be a threshold in $\dot{M}_{\mathrm{d}}(t) /\left\langle\dot{M}_{\mathrm{d}}(t)\right\rangle$. It is quite difficult to establish whether this hypothesis is correct. In the positive, one has to explain what produces the hard tail: the physical process should be strongly dependent on what happens in the disk. When the hard X-ray component is no longer detected (regions II, III and IV of the HID), we observe a slight decrease of the temperature of the scattering cloud, as the source moves from the left to the right in the HB. In the van der Klis (2001) model, this is related to the variation of $\dot{M}$ : a higher prompt disk accretion rate is expected to push the inner radius towards the neutron star with a consequent larger production of seed and hotter photons for the corona, which is thus more efficiently Compton-cooled ( $k T_{\mathrm{e}}$ decreases from regions II to IV, see Table 4$)$. We confirm the presence of the $\mathrm{Fe} \mathrm{K}$ emission line at $\sim 6.7 \mathrm{keV}$, while the edge detection (first reported by DS2000) is not so evident and should be treated carefully. Indeed we find that the EQPAIR model well fits the data (see Table 3) and neither Comptonreflection nor absorption edge are required, showing that the presence of such components in the spectra are very sensitive to the continuum model adopted. One should be careful in claiming the presence of these components in the case of GX 17+2.

\section{Conclusions}

We have investigated two BeppoSAX observations of the Z source GX 17+2 performed on 1997 April 3 and 21. The persistent continuum X-ray emission can be described by two-component models, which support the well known accretion scenarios suggested by the Western and Eastern model. However, in the first observation, when the source was in the upper left HB of the HID diagram, we observed a hard X-ray tail (up to $120 \mathrm{keV}$ ), which disappeared in the second observation performed two weeks later, when the source moved to the right in the HB. Our results confirm those obtained by DS2000, but provide a stronger evidence for the presence of a threshold in the accretion rate $\dot{M}$ in the HB, above which the intensity of the hard tail is below the instrument sensitivity or disappears. Given that the X-ray luminosity is not correlated with the position of the source in the HID, $\dot{M}$ is not the one we ascribe to X-ray luminosity but has a somewhat different meaning. We propose as working hypothesis the model by van der Klis (2001), in which the X-ray luminosity is proportional to the prompt disk accretion rate plus its own long-term average, while motion along the $\mathrm{Z}$ track depends on the ratio of these two quantities. To testify whether this hypothesis is correct, more observational and theoretical work is required. The hard X-ray excess observed in the first observation can be described by Comptonization of soft seed photons $\left(k T_{\mathrm{s}} \sim 1 \mathrm{keV}\right)$ off non-thermal electrons injected with spectral index $p \sim 1.7$. In addition, a thermal component described by a BB with $k T_{\mathrm{bb}} \sim 0.6 \mathrm{keV}$ is observed. An emission line at $\sim 6.7 \mathrm{keV}$ is found in both observations. The origin of the non-thermal electrons cannot be established on the basis of the BeppoSAX data alone. We have two equally acceptable options: electrons from jets or electrons produced in magnetohydrodynamic turbulence processes occurring in the corona. Only the determination of the source spectrum at higher energies $(\gtrsim 200 \mathrm{keV})$ can provide more constraints on the origin of the high-energy excess. A step forward could be done by INTEGRAL through a long monitoring observation of the source. Also high-energy resolution observations below $10 \mathrm{keV}$, performed with Chandra or $X M M-N e w t o n$, could be very helpful in clarifying the origin of the $\mathrm{Fe}$ emission line and to determine unambiguously the presence/absence of an absorption edge. On the other hand, simultaneous X-ray/radio observations would test the possibility of a correlation between hard X-ray and radio emission in this source, with presence or not of relativistic expanding lobes.

Acknowledgements. This research is supported by the Italian Space Agency (ASI) and Ministry of University and Scientific Research of Italy (PRIN N. 2002027145). A.A.Z. has been supported by KBN grants PBZ-KBN-054/P03/2001 and 1P03D01827.

\section{References}

Anders, E., \& Ebihara, M. 1989, Geochim. Cosmochim. Acta, 46, 2363

Asai, K., Dotani, T., Mitsuda, K., et al. 1994, PASJ, 46, 479

Augusteijn, T., Karatasos, K., Papadakis, M., et al. 1992, A\&A, 265, 177

Barret, D. 2001, Adv. Space Res., 28, 307

Barret, D., \& Olive, J. 2002, AJ, 576, 391

Boella, G., Butler, R. C., Perola, G. C., et al. 1997a, A\&AS, 122, 299

Boella, G., Chiappetti, L., Conti, G., et al. 1997b, A\&AS, 122, 327

Chiappetti, L., \& Dal Fiume, D. 1997, in Proceedings of the Fifth International Workshop on Data Analysis in Astronomy System, ed. V. di Gesù, M. J. B. Duff, A. Heck, M. C. Maccarone, L. Scarsi, \& H. U. Zimmerman (World Scientific Press), 101

Coppi, P. S. 1999, in High Energy Processes in Accreting Black Holes, ed. J. Poutanen, \& R. Svensson, ASP Conf. Ser., 161, 375

Crosby, N., Vilmer, N., Lund, N., \& Sunyaev, R. 1998, A\&A, 334, 299

D’Amico, F., Heindl, W. A., Rothschild, R. E., \& Gruber, D. E. 2001, ApJ, 547, L147

Di Salvo, T., Stella, L., Robba, N. R., et al. 2000, ApJ, 544, L119 (DS2000)

Di Salvo, T., Robba, N. R., Iaria, R., et al. 2001, ApJ, 554, 49 
Di Salvo, T., Farinelli, R., Burderi, L., et al. 2002, A\&A, 386, 535

Di Salvo, T., \& Stella, L. 2002 [arXiv: astro-ph/0207219]

Fender, R. 2002, in Relativistic outflows in Astrophysics, ed. A. W. Guthmann, M. Georganopoulos, A. Marcowith, \& K. Manolakou, Lecture Notes in Physics, 589, 101 [arXiv: astro-ph/0109502]

Fiore, F., Guainazzi, M., \& Grandi, P. 1999, Technical Report 1.2, BeppoSAX scientific data center, available online at ftp://ftp.asdc.asi.it/pub/sax/doc/software_docs/ saxabc_v1.2.ps

Friedman, H., Byram, E. T., \& Chubb, T. A. 1967, Science, 156, 374

Frontera, F., Costa, E., Dal Fiume, D., et al. 1997, A\&AS, 122, 357

Frontera, F., Dal Fiume, D., Malaguti, G., et al. 1998, The Active X-ray Sky, ed. L. Scarsi, H. Bradt, P. Giommi, \& F. Fiore, Nuclear Physics, Proc. Suppl. 69/1-3, 286

Frontera, F., Palazzi, E., Zdziarski, A. A., et al. 2001, ApJ, 546, 1027

Gierliński, M., Zdziarski, A. A., Poutanen, J., et al. 1999, MNRAS, 309,496

Gierliński, M., \& Done, C. 2003, MNRAS, 342, 1083

Hasinger, G., van der Klis, M., Ebisawa, K., Dotani, T., \& Mitsuda, K. 1990, A\&A, 235, 131

Hertz, P., Vaughan, B., Wood, K. S., et al. 1992, ApJ, 396, 201

Homan, J., van der Klis, M., Jonker, P. G., et al. 2002, ApJ, 568, 878

Hoshi, R., \& Asaoka, I. 1993, PASJ, 45, 567

Iaria, R., Burderi, L., Di Salvo, T., La Barbera, A., \& Robba, N. R. 2001, ApJ, 547, 412

Kahn, S. M., \& Grindlay, J. E. 1984, ApJ, 281, 826

Kuulkers, E., \& van der Klis, M. 1996, A\&A, 314, 567

Magdziarz, P., \& Zdziarski, A. A. 1995, MNRAS, 273, 837

Manzo, G., Giarrusso, S., Santangelo, A., et al. 1997, A\&AS, 122, 341

McClintock, J. E., \& Remillard, R. A. 2004, in Compact Stellar X-ray Sources, Chap. 4 , in press, ed. W. H. G. Lewin, \& M. van der Klis (Cambridge University Press) [arXiv: astro-ph/0306213]
Mitsuda, K., Inoue, H., Koyama, K., et al. 1984, PASJ, 36, 741

Mitsuda, K., Inoue, H., Nakamura, N., \& Tanaka, Y. 1989, PASJ, 41, 97

Parmar, A. N., Martin, D. D. E., Bavdaz, M., et al. 1997, A\&AS, 122, 309

Penninx, W., Lewin, W. H. G., Zijlstra, A. A., Mitsuda, K., \& van Paradijs, J. 1988, Nature, 336, 146

Piraino, S., Santanelo, A., \& Kaaret, P. 2002, ApJ, 567, 1091

Sunyaev, R. A., \& Titarchuk, L. 1980, A\&A, 86, 121

Sztajno, M., van Paradijs, J., \& Lewin, W. H. G. 1986, MNRAS, 222, 499

Tawara, Y., Tatsumi, H., \& Tsuneo, K. 1984, PASJ, 36, 861

Titarchuk, L. G. 1994, ApJ, 434, 570

van der Klis, M. 2001, ApJ, 561, 943

van Paradijs, J., Allington-Smith, J., Callanan, P., et al. 1990, A\&A, 235, 156

Vrtilek, S. D., Raymond, J. C., Garcia, M. R., et al. 1990, A\&A, 235, 162

Vrtilek, S. D., Penninx, W., Raymond, J. C., et al. 1991a, ApJ, 376, 278

Vrtilek, S. D., McClintock, J. E., \& Seward, F. D. 1991b, ApJS, 76, 1127

White, N. E., Mason, K. O., \& Sanford, P. W. 1978, ApJ, 220, 600

White, N. E., Peacock, A., Hasinger, G., et al. 1986, MNRAS, 218, 129

White, N. E., Stella, L., \& Parmar, A. N. 1988, ApJ, 324, 363

Zdziarski, A. A., Lightman, A. P., \& Maciołek-Niedźwiecki, A. 1993, ApJ, 414, L93

Zdziarski, A. A., Grove, J. E., Poutanen, J., Rao, A. R., \& Vadawale, S. V. 2001, ApJ, 554, L45

Zdziarski, A. A., \& Gierliński, M. 2004, Progr. Theor. Phys. Suppl., 155, 99 